

\section{Primary ciliary dyskinesia: a major player in a bigger game}

Primary ciliary dyskinesia (PCD) is an inherited disorder of clinical and genetic heterogeneity resulting from mutations in genes involved in the transport, assembly and function of motile cilia. The resulting impairment in mucociliary clearance means patients suffer from chronic progressive lung disease, bronchiectasis, rhinosinusitis and middle ear disease. Subfertility is common to both male and female patients. Situs abnormalities occur in around half of patients, with a subgroup suffering more complex situs arrangements where congenital heart defects or other organ abnormalities frequently coexist. Variations from the classical PCD phenotype are increasingly recognised where overlapping features across a range of motile and nonmotile ciliopathies are redefining our approach to both diagnosis and management of these complex conditions. PCD offers an ideal opportunity for direct visualisation of ciliary function and structure, following nasal brush biopsy, allowing opportunities for researchers to directly interrogate the downstream impact of loss of function mutations. In turn, this has led to rapid advances in the development of new diagnostic tests. These advances mean that PCD is an excellent disease model for understanding the genetic and mechanistic causes of the clinical phenotype for all respiratory ciliopathies. Furthermore, the overlapping role of motile ciliary defects in a wider set of complex and syndromic disorders related to loss of function mutations in primary, nonmotile cilia has been recognised. As we better understand the role of ciliary defects in a broad spectrum of diseases, we should aim to map out a framework through which we can identify, diagnose and treat all respiratory ciliopathies.

\section{Ciliopathies: understanding the role of cilia in human health and disease}

Cilia are organelles that project from the surface of most cells within the body, playing an important role in many regulatory processes including cell signalling, reproduction, development, organogenesis and as a primary defence mechanism

@ERSpublications

Primary ciliary dyskinesia is an inherited disorder of the motile cilia, and an excellent model to examine the structure, function and heterogeneity of a wider group of ciliopathies where overlapping phenotypes are recognised https://bit.ly/3dLb7as in some organs [1-3]. Throughout evolution, the function and structure of cilia has been preserved, and they are found on nearly all vertebrate cells [4]. It is helpful to differentiate between the two categories of cilia, primary (nonmotile) and motile cilia. Primary cilia are single, nonmotile organelles on the surface of almost all cells in the human body that act as sensors picking up chemical and physical stimuli, moderating signal pathways in the environment around them [2].
Cite as: Bhatt $\mathrm{R}, \operatorname{Hogg} \mathrm{C}$. Primary ciliary dyskinesia: a major player in a bigger game. Breathe 2020; 16: 200047. 
Primary cilia determine organogenesis and normal development [5], and so loss of function mutations in nonmotile cilia genes result in a group of rare syndromic disorders [6]. In contrast, motile cilia are present on a group of specialised epithelial surfaces, usually several hundred to a cell, and act as a mucociliary escalator moving fluid and debris in a synchronised and unidirectional way at the air-liquid interface [3, 7]. Multiple motile cilia are located throughout the airway and sinuses, Eustachian tube, brain ependyma, fallopian tubes and as a single cilium in the sperm tail. The exception to the rule for motile cilia are the nodal cilia. Here, single motile cilia on the cells within the embryonic node have a different axonemal structure with a circular waveform resulting in leftward flow of liquid and calcium across the node during organogenesis. This transient function in the developing embryo determines organ laterality and is often known as the left-right organiser [8]. Loss of function mutations in motile cilia genes result in multisystem disorders potentially impacting on lung health, fertility, organ situs, congenital heart defects and the normal circulation of spinal fluid [9].

Collectively, these inherited disorders of human cilia are called ciliopathies. In most cases, these rare genetic conditions affect either primary cilia resulting in syndromic ciliopathies, or motile cilia such as in primary ciliary dyskinesia (PCD). These previously disparate disorders are now recognised as part of a ciliopathy spectrum where an overlapping clinical phenotype may be evident [4, 10]. Variation in the severity of syndromic features suggests that, in some cases, where overlapping phenotypes exist, genetic modifiers determining disease expression come into play [11]. Individually and combined, >35 inherited ciliopathy diseases are known, resulting from dysfunction of primary or motile cilia, or both. Globally, one in 2000 individuals are affected with significant comorbidity, challenging healthcare systems to improve diagnostics and management for patients with these complex multisystem conditions [10].

\section{A broader perspective of respiratory ciliopathies}

Ciliary dysfunction, in motile and nonmotile cilia, leads to a wide spectrum of clinical phenotypes that may overlap genetically and phenotypically. Cilia play an important role in organ function and development, with an increasing recognition of the complex conditions associated with ciliary defects [12]. These defects manifest in a variety of ciliopathy syndromes that have a different set of cardinal signs from patients with PCD that include polydactyly, visual and hearing defects, obesity, and renal and cardiac defects. Loss-of-function mutations in primary and motile cilia were thought to have distinct phenotypes relating to sensory or motor functions, respectively. However, it has become clear that motile cilia in the airway also have a sensory role $[13,14]$ and that defects in certain ciliary proteins, that are important for the function of both motile and nonmotile cilia, can lead to a range of ciliopathy phenotypes with overlapping clinical features. For example, patients with Bardet-Biedl syndrome (BBS), when investigated for a high background prevalence of respiratory PCD-like symptoms (neonatal respiratory distress, rhinitis and otitis media), had some curious diagnostic findings. These patients were found to have normal structure of the motile cilia but with some unique inclusions near the tip of the ciliary axoneme; however, function was apparently preserved [15]. Similar findings have been recorded in other ciliopathy syndromes, where long, whip-like cilia and inclusion bodies near the axonemal tip, like those seen in the BBS cohort, have become a recognised feature in a subgroup of patients where an overlapping respiratory phenotype exists. Loss of function mutations in $R P G R$, causing progressive blindness in patients with retinitis pigmentosa (RP), are also known, although rare, mutations leading to a syndromic PCD-RP phenotype. Like in BBS, in these patients, assessment of ciliary structure using transmission electron microscopy (TEM) was normal but with disruption of ciliary orientation leading to a functional defect [16]. A further example of phenotypic overlap is seen in patients with autosomal dominant polycystic kidney disease (ADPKD). Polycystin 1 and 2, the protein abnormalities associated with ADPKD, are also found in cilia and smooth muscle cells in the airway, implicating their role in an increased prevalence of bronchiectasis in these patients $[17,18]$. Finally, a small group of patients with a de novo mutation in FOXJ1 presenting with a collection of clinical anomalies related to defects in motile cilia (hydrocephalus, bronchiectasis and randomisation of left-right asymmetry) appear to have a heterozygous autosomal dominant mode of inheritance [19]. Diagnostic tests, including highspeed video microscopy, immunofluorescence (IF) and TEM, indicate paucity of cilia with mislocalisation of the basal bodies. Specific staining for protein tyrosine kinase 2, a focal adhesion protein, confirms aberrant localisation in the cytoplasm of the respiratory epithelial cells in patients with this mutation [19]. This group of patients represent the first reported cases of a dominantly inherited motile ciliopathy, further highlighting the complexity of this heterogeneous group of disorders

Importantly, syndromic patients that are referred with symptoms in keeping with a respiratory ciliopathy should undergo the diagnostic pathway that is in place for PCD to confirm or exclude functional abnormalities related to concomitant defects of the motile cilia. However, when seeing patients who have a strong history for PCD but do not follow the "norm", we should consider the presence of other ciliopathies. 


\section{PCD as a model for ciliopathies}

PCD is a heterogeneous multisystem disorder of motile cilia leading to a common clinical phenotype of rhinosinusitis, middle ear disease, bronchiectasis and subfertility. Situs abnormalities occur in almost half of patients, and associated congenital heart diseases and other organ abnormalities occur in a subgroup of patients with situs ambiguus or heterotaxy [20, 21]. PCD is the most common and well phenotyped of the respiratory ciliopathies, with clearly defined functional and structural defects that can be determined from an array of tests across a comprehensive, but not fail-proof, diagnostic algorithm. Despite rapid advances bringing new diagnostic tools from bench to bedside [22, 23], gaps in our knowledge remain. A greater understanding of the underlying disease mechanisms and genetics, where next-generation sequencing has advanced gene discovery with lossof-function mutations in more than 50 respiratory ciliopathy genes now reported [24]. Increased knowledge of cilia genes and observed functional defects in a wider array of ciliopathy conditions is challenging perceptions of the clinical phenotype, where overlapping features between motile and nonmotile ciliopathies are emerging. PCD, as a disease, offers clinical scientists a unique research model with the opportunity to examine both the structure and function of motile cilia directly from the airway epithelium, or following regeneration of ciliated epithelium in cell culture [25]. It has, therefore, been possible to interrogate the downstream impact of novel gene variants, determining pathogenicity and expanding our knowledge of a wider set of ciliopathy syndromes.

\section{Diagnostic tests: state-of- the-art algorithms and basic rules to secure a diagnosis}

The diagnosis of PCD involves a complex, timeconsuming and expensive diagnostic pathway that has determined that reliable testing is only available or practical in specialist centres where expertise has grown around a clinical academic interest in this rare condition. Separate diagnostic guidelines have been published by the European PCD Consortia [26, 27] and their North American counterparts [28], leading to controversy around which tests, and how many, should be employed to optimise diagnostic accuracy. A welcome European-North American review of the guidelines has outlined why different approaches to PCD diagnostics has arisen, clarifying why a uniform diagnostic algorithm across all reference centres is a challenge from a pragmatic perspective [29]. This review will not debate that issue but aims to outline the diagnostic tests available that, in various combinations, can be used in a targeted cascade to optimise diagnostic potential in the majority of cases (figure 1). The take home message is that to diagnose respiratory ciliopathies, including PCD, rarely does a single test suffice. In particular, as we expand our expertise across a wider group of ciliopathy conditions, a robust approach is required to ensure the functional and structural findings are consistent with candidate gene variants to support diagnostic confidence.

Historically, diagnosis has relied on determining the function and structure of motile cilia by using high-speed video microscopy (HSVM) and TEM, respectively. Genotyping and novel imaging techniques, moving in from the research environment, offer improved accuracy when used in combination with HSVM and TEM, and include immunofluorescent antibody testing (IF) and super-resolution three-dimensional (3D) electron tomography imaging.

The majority of cases do not need the full panel of tests to be deployed. Rather, a staged approach is appropriate to ensure a clear diagnosis is made, or indeed excluded. Clinical assessment is key to deciding which cases need referral for testing and having some basic rules or a scoring system, such as PICADAR [30], can be helpful. If a patient exhibits some or all of the cardinal signs - situs abnormalities, unexplained neonatal respiratory distress, persistent rhinitis from early life, middle ear disease with complications such as otorrhoea post-grommet surgery, or congenital heart disease - then they warrant referral to a PCD centre where the following screening and diagnostic tests might be applied.

\section{Nasal nitric oxide}

The measurement of nasal nitric oxide ( $\mathrm{nNO}$ ) represents an accurate and immediate screening tool for patients $>5$ years of age [31]. It has largely replaced the more archaic techniques using saccharine or charcoal to assess ciliary function in the nose [32] and, in centres with a chemiluminescent analyser and specialist team to undertake accurate and routine $\mathrm{NO}$ measurements, plays an invaluable role in the diagnostic pathway. Limitations relate to patient cooperation, and other causes of nasal obstruction or conditions where lower $\mathrm{nNO}$ measurements are characteristic, such as in cystic fibrosis (CF) [33]. Recently, it has also been recognised that some respiratory and many syndromic ciliopathies have normal $\mathrm{nNO}$ and, in these cases, the pursuit of a diagnosis must continue with the implementation of additional diagnostic testing [34]. For a screening tool to fulfil its potential, it would ideally be widely available to clinicians. The quest for a portable nNO device that matches the accuracy of the chemiluminescent analysers based at reference centres continues. NIOX, with the MINO [35] and, more recently, the VERO [36] device, have provided the potential for screening patients in a wider setting that should result in direct referrals to a reference centre if 


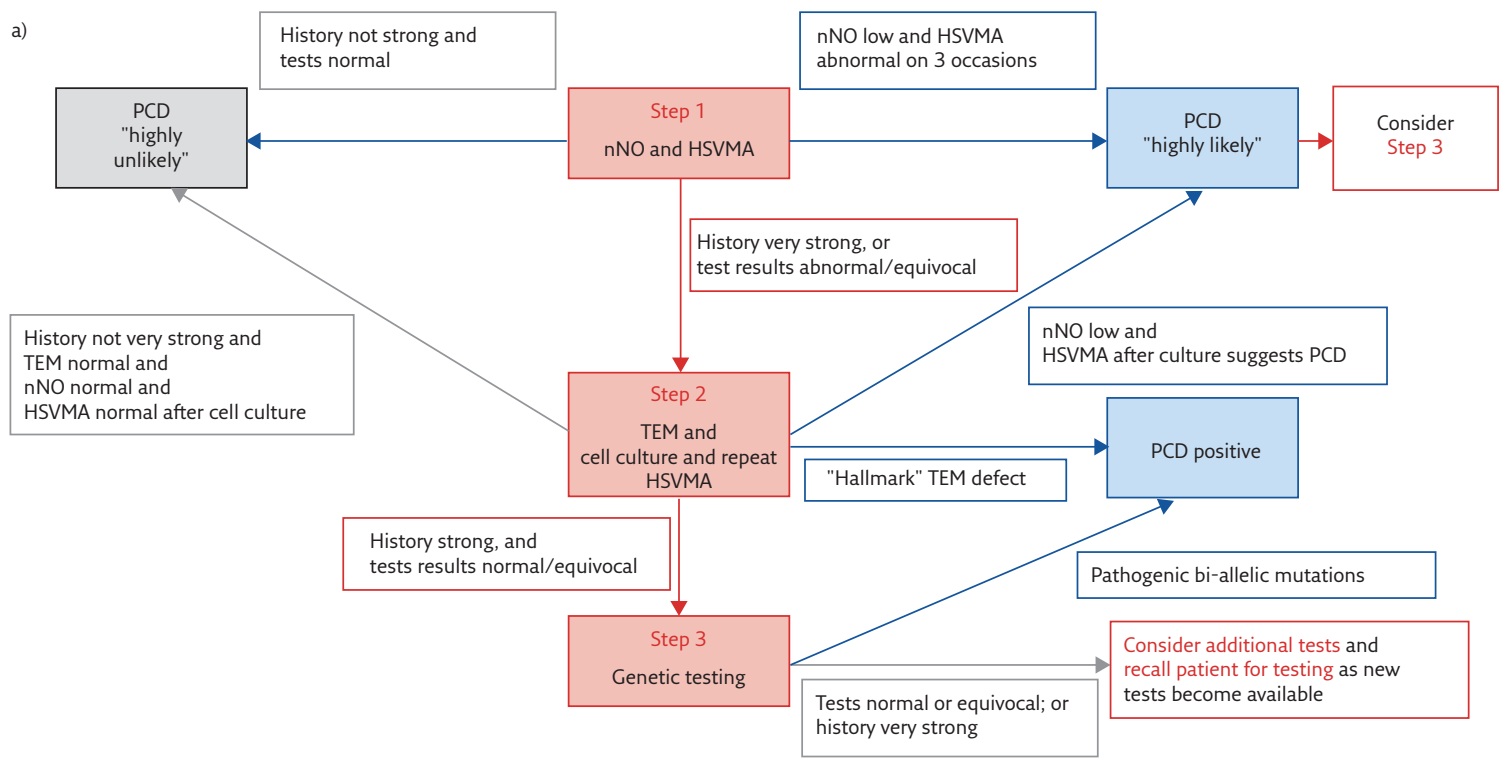

b)

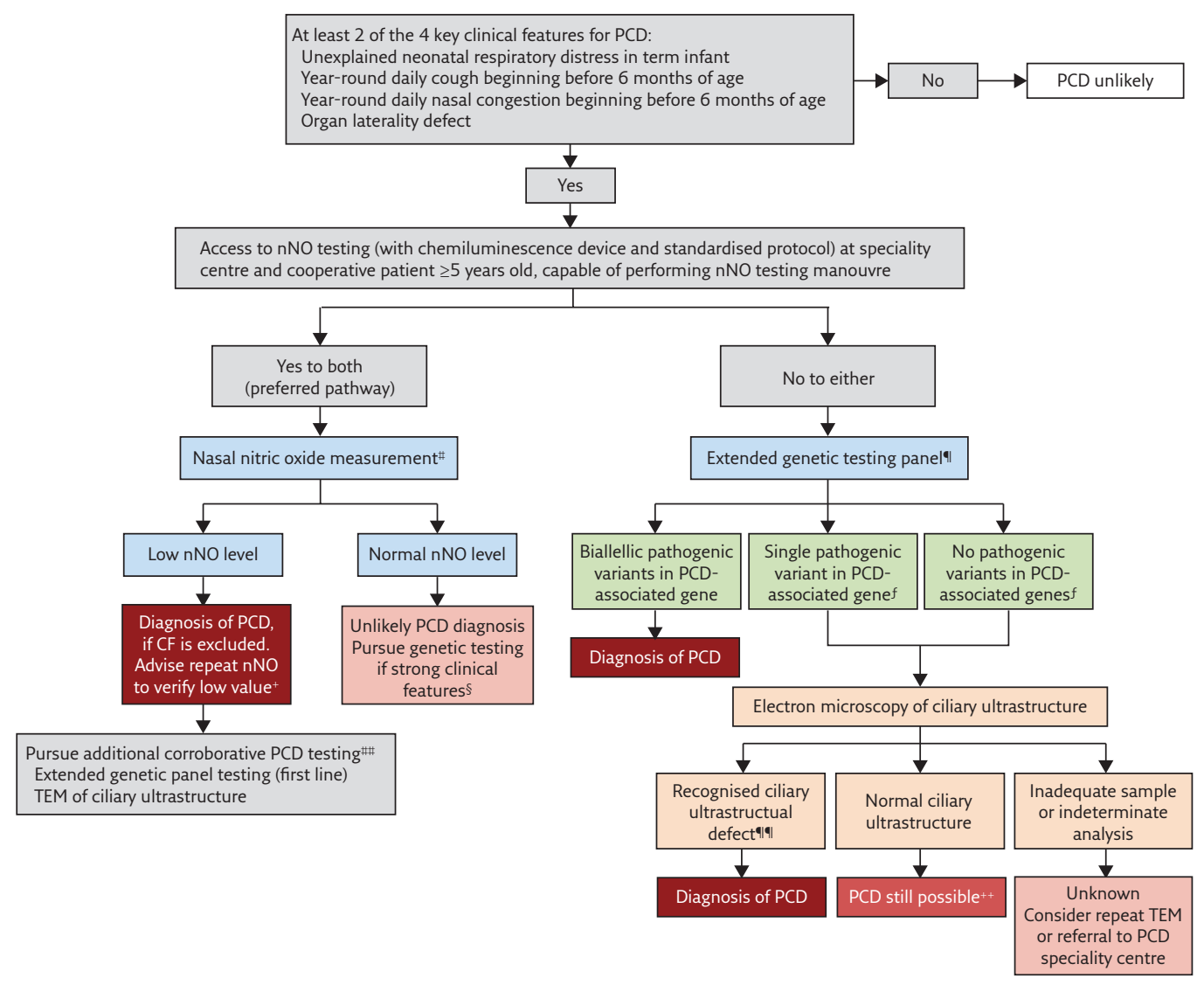

Figure 1 Flowchart to demonstrate the pathways to diagnosis of PCD as per the a) European Respiratory Society and b) American Thoracic Society consensus statements. It is important to note that not all patients will require all investigations. \#: cystic fibrosis (CF) should be ruled out before performing nasal nitric oxide ( $n N O)$ measurement, as roughly one-third of CF patients can have $n N O$ values below PCD diagnostic cut-offs. nNO measurements should only be performed with chemiluminescence analysers using standardised protocols at centres with specific expertise in nNO measurements. Some nNO analysers have not received approval from federal agencies worldwide (US Food and Drug Administration and Health Canada), which may have implications for clinical implementation. "I: genetic panel testing for mutations in $>12$ disease-associated PCD genes, including deletion/duplication analysis. ${ }^{+}:$as $n N O$ levels can be significantly decreased by viral respiratory tract infections, a repeat $n N O$ measurement, at least 2 weeks after the initial low value (expert opinion), is recommended to ensure the initial low value is not secondary to a viral process. A normal $n N O$ value upon repeat testing is not suggestive of $P C D$, as $n N O$ values remain consistently low in $P C D$. s: most forms of $P C D$ resulting in normal nNO levels have normal or non-diagnostic electron microscopy studies. Thus, genetic testing is recommended in these cases. f: or presence of variants of unknown significance. \#! additional corroborative testing may provide information on clinical prognosis, further disease understanding, and potential future therapeutic considerations. 9/9: known disease-associated TEM ultrastructural defects include outer dynein arm (ODA) defects, ODA plus inner dynein arm (IDA) defects, IDA defect with microtubular disorganisation, and absent central pair, identified using established criteria. Of note, the presence of IDA defects alone is rarely diagnostic for $P C D .{ }^{++}$: up to $30 \%$ of PCD cases can have normal ciliary ultrastructure on electron microscopy. Consider referral to PCD specialty centre if there is a strong clinical phenotype but all electron microscopy and genetic testing are negative. HSVMA: high-speed video microscopy analysis. Reproduced from [29] with permission. 
clinical phenotype and nNO measurements raise the possibility that a patient may have a respiratory ciliopathy.

\section{Ciliary beat pattern and frequency}

Despite not being diagnostic in its own right, ciliary function, measured by beat pattern and frequency using HSVM, remains consistently the most abnormal test in confirmed cases of PCD [37]. However, as a technique, it requires significant experience to develop accuracy in the interpretation of a complex and growing array of specific beat patterns, and so it is not always available in the clinical diagnostic algorithm at some reference centres. In expert hands, it is an invaluable tool where recognised beat pattern abnormalities are correlated with specific structural defects and genetic mutations, and in some cases, consistent beat pattern anomalies have led to the pursuit of a difficult diagnosis where TEM or genetic evidence was elusive [23] (table 1).

\section{Ciliary structural analysis}

In contrast, the determination of hallmark structural defects using TEM (table 1) is a recognised diagnostic test, albeit it with an accurate pick-up in only $75-80 \%$ of cases [38]. Using TEM to determine loss of specific proteins within the ciliary axoneme was considered the gold standard to secure a diagnosis (figure 2) but it is increasingly recognised that in some cases where a clear clinical phenotype was present, TEM was normal. Like all PCD diagnostic tests, TEM should be used as part of a wider set of investigations.

\section{Immunofluorescent antibody staining of ciliary proteins}

IF staining represented the first real opportunity to develop a reliable, cost-effective test within the PCD diagnostic pathway that can potentially be used in a wider laboratory setting. IF has long been used in PCD genetic research [39] to determine localisation of protein compounds within the ciliary axoneme, proving instrumental in confirming specific mutations as pathogenic variants. IF also offers value in clinical diagnostics where an established antibody panel has been validated using antibodies for specific proteins identifiable using TEM [22]. Additional fluorescent antibodies are now in clinical use helping to bridge diagnostic gaps when using TEM, such as in cases of DNAH11

Table 1 Beat pattern assessment using HSVM, and correlations with TEM structural phenotype and genotype

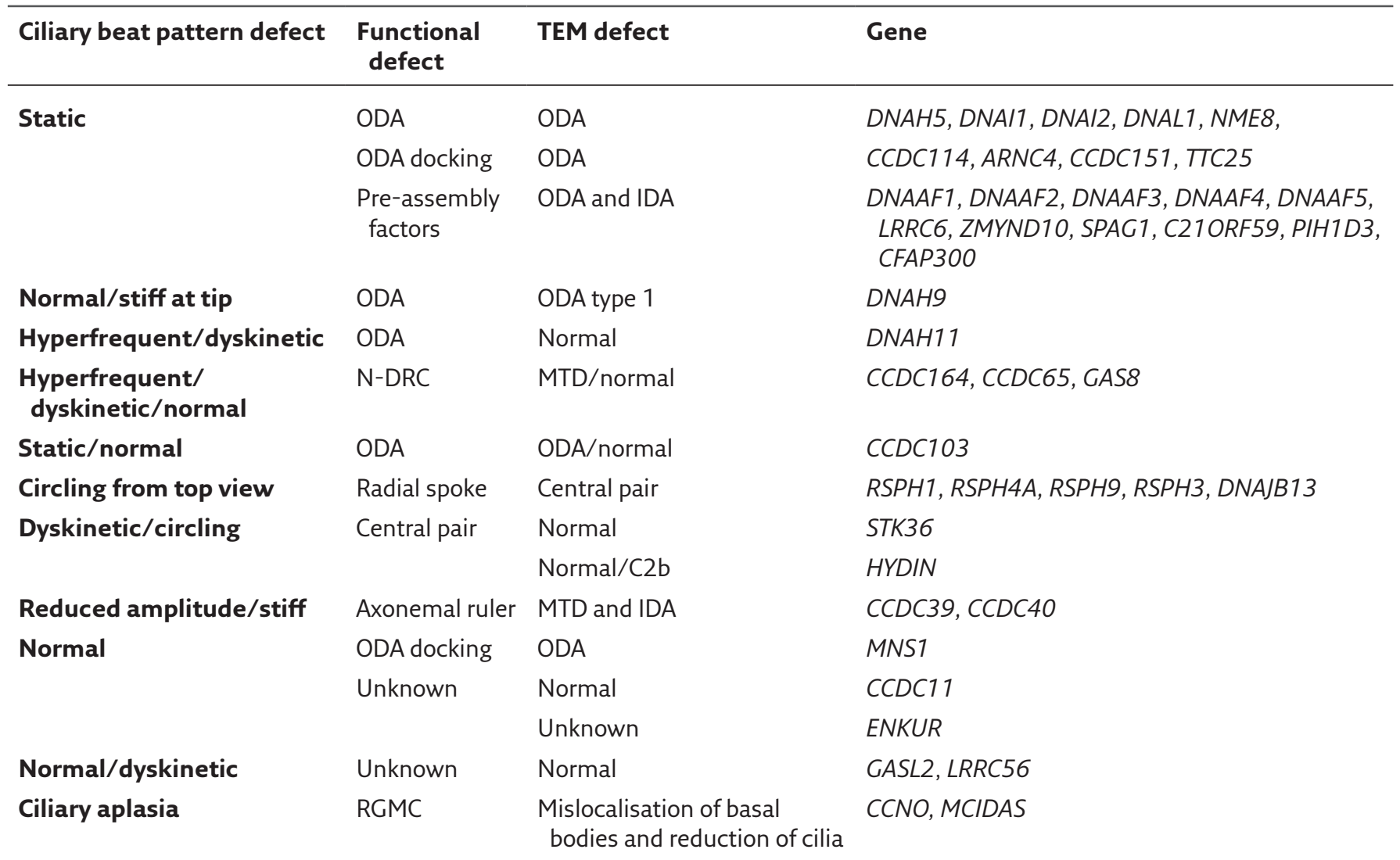

ODA: outer dynein arm; IDA: inner dynein arm; N-DRC: nexin-dynein regulatory complex; MTD: microtubular disorganisation; RGMC: reduced generation of multiple motile cilia. 

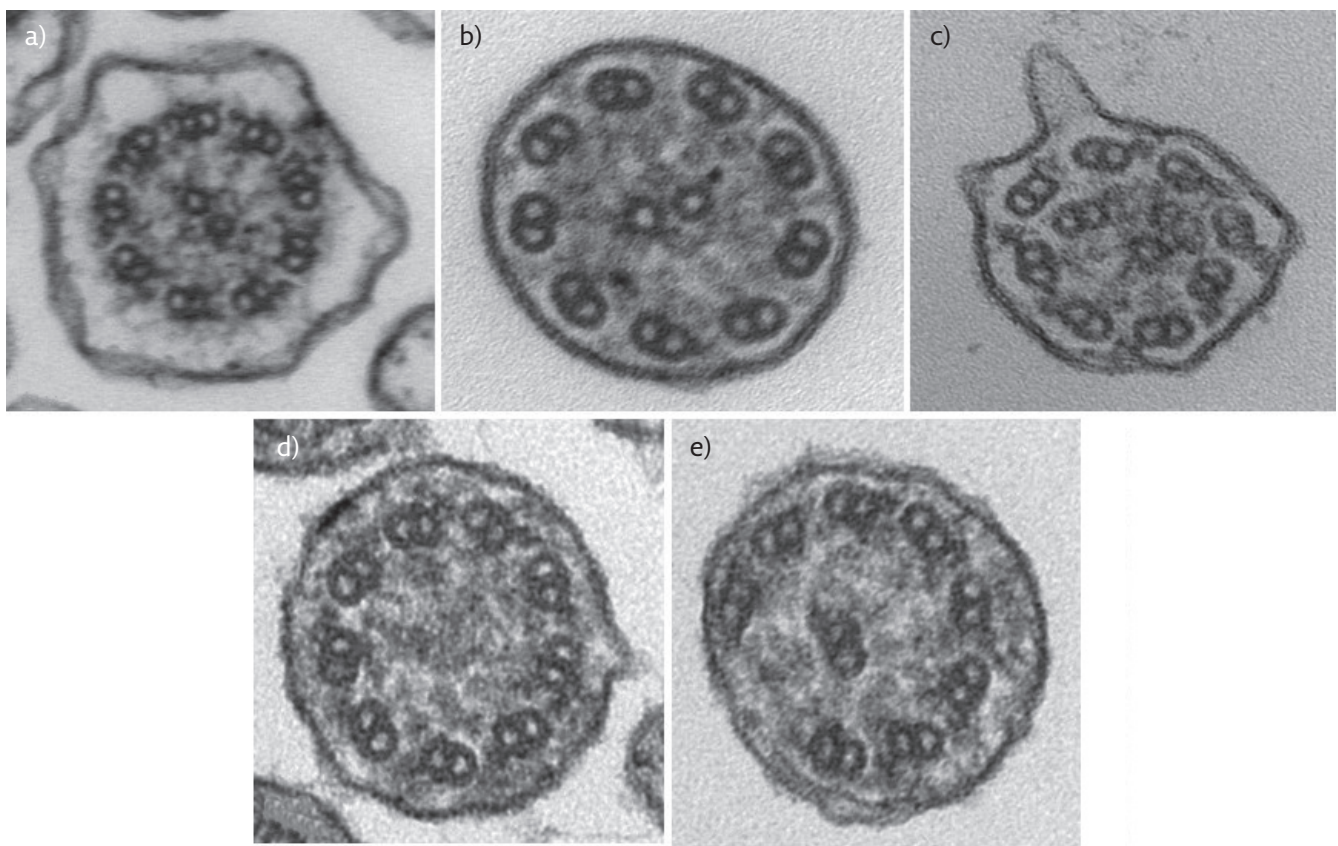

Figure 2 TEM images of cross-sections of cilia from nasal brushing samples. a) Normal; b) outer and inner dynein arm defects; c) inner dynein arm defects plus microtubular disorganisation; d) central pair defect; and e) transposition defect.

mutation (figure 3), adding further value to its use in the clinical setting. This test is now utilised in most reference centres and continues to play a key role in genetic research laboratories engaged in novel variant analysis of candidate genes.

\section{D electron tomography}

This technique uses a tilt series of electron micrograph images that are then aligned and stacked to generate $3 \mathrm{D}$ super resolution rendered reconstructions of cilia giving detailed ultrastructural information not visible using TEM. Although largely a research tool, its application clinically has led to confirmation of difficult cases, determination of pathogenic mutations and a better understanding of protein localisation within the axoneme. HYDIN mutation is an excellent example where tomography demonstrated loss of the protein, $\mathrm{C} 2 \mathrm{~b}$, in the central pair apparatus, confirming the downstream deleterious effect of the mutation. Defects using TEM for patients with HYDIN mutation have been difficult to detect, but easily visualised using tomography [40] (figure 4). Correlation with genetic variants and beat pattern anomalies has led to a growing confidence in the recognition of subtle TEM defects in the central pair that may previously have been dismissed. Likewise, tomography has played a key role in the determination of DNAH1 1, where typically patients have low $\mathrm{nNO}$ and a stiff hyperkinetic beat pattern but normal TEM findings. Tomography clearly identifies loss of the DNAH11 protein in the proximal part of the outer dynein arm (ODA), correlating with the proximal stiffness on beat pattern analysis using HSVM [41] (figure 5). Patients with DNAH11 mutation are now easily diagnosed using both genetics and IF staining but it is an example where experts consistently identified beat pattern anomalies using HSVM, recognising that

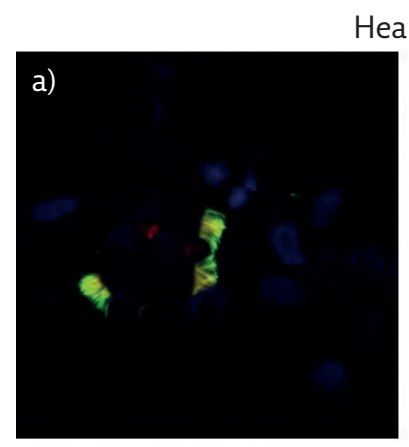

Healthy control

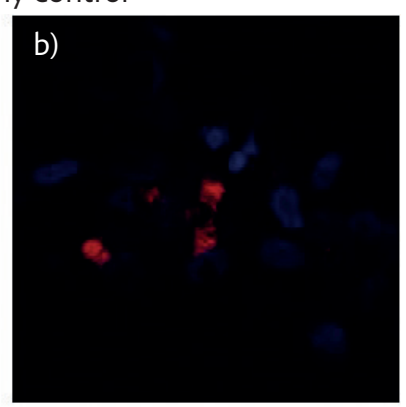

Patient with DNAH11 mutation
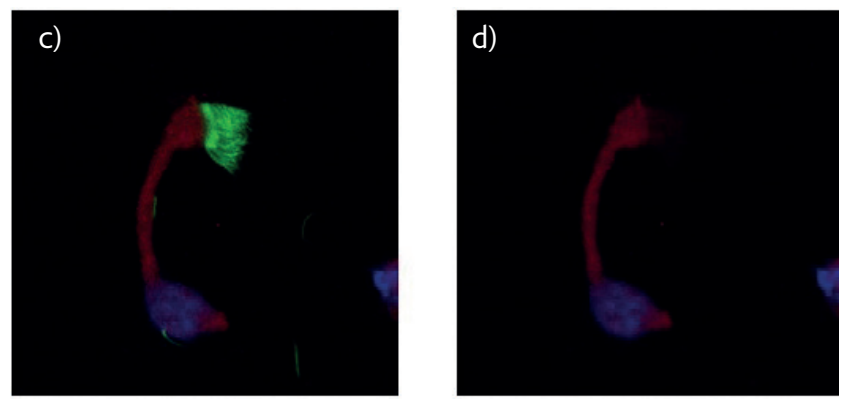

Figure 3 IF staining in a healthy control where a) tubulin stains green to indicate presence of cilia on the cell surface, and b) staining with DNAH5 antibody for the outer dynein arm (ODA) in red is identical, indicating normal ODA structure; and in c) and d) a patient with DNAH11 mutations, where c) tubulin staining green for cilia is seen whereas in d) absence of staining for DNAH11 using red labelled antibody is evident. 

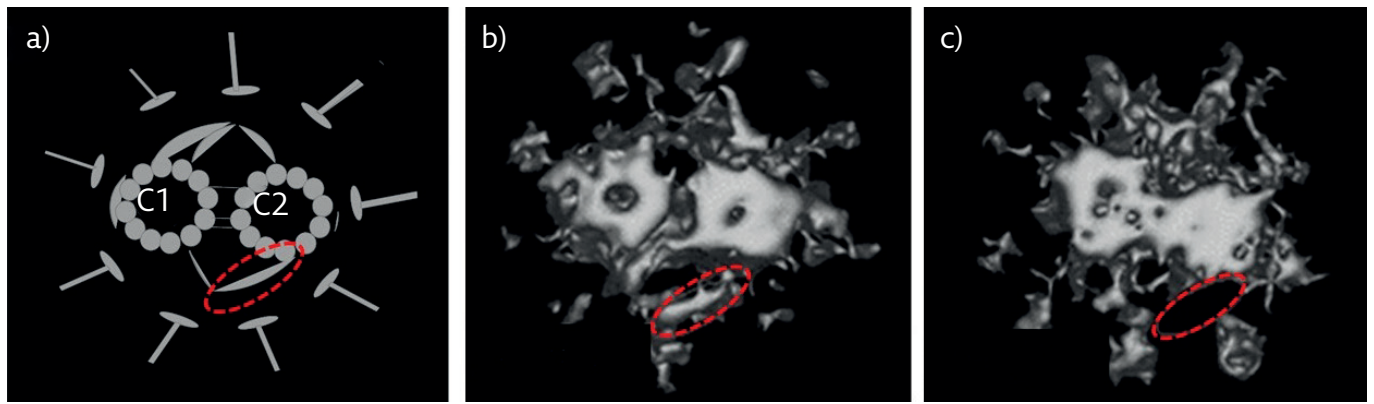

Figure 43 D electron tomography series demonstrating the clear difference between the healthy control sample and that from a patient with HYDIN mutation. a) Schematic interpretation of the central pair of a normal cilia highlighting the $C 2 b$ protein that is absent in patients with HYDIN (red circle). b) 3D tomogram of healthy control showing normal appearance of $C 2 b$ in the central complex and c) $3 D$ tomogram from a patient with HYDIN showing absence of $C 2 b$.

this cohort were highly likely to have PCD before the techniques described here were available to confirm pathogenic variants in DNAH11.

\section{Genotyping}

The advance in genome sequencing has had significant impact on the diagnosis of PCD. To date, mutations in at least 50 genes have been identified as pathogenic in PCD [24, 42, 43]. Biallelic recessive, hemizygous $X$-linked and, more recently, heterozygous de novo dominant [19] pathogenic mutations in known ciliopathy genes are accepted as diagnostic, but around $20-25 \%$ of cases cannot be confirmed using genetic testing. Genes encoding ciliary structure and function in most cases correlate with the findings on TEM, IF and HSVM. However, in many cases, VUS (variants of unknown significance) require diagnostic confirmation using one or more of these tests to avoid misdiagnosis where missense nonpathogenic variants are identified, and all genetic diagnoses must be supported in this way [42].

\section{Heterogeneity and disease mechanisms}

The clinical impact of mutations affecting motile cilia results from disruption of the normal mucociliary escalator with mutation specific phenotypic variations. Genotypic-phenotypic correlations have shown some mutations are not associated with laterality defects, others more likely to affect fertility, have a higher prevalence of hydrocephalus or affect the severity of pulmonary disease [21, 24, 44-46].

Understanding the spectrum of clinical symptoms and disease correlations requires some knowledge of the underlying disease mechanisms and where specific mutations impact ciliary development. Table 2 outlines how the 50 known mutations fall into categories impacting preassembly, assembly, transport or docking of ciliary proteins, and where clear correlations have been identified. For instance, it is recognised that defects in genes encoding ODA components, assembly and docking as well as the proteins anchoring the dynein regulatory complex are associated with randomisation of left-right asymmetry or situs

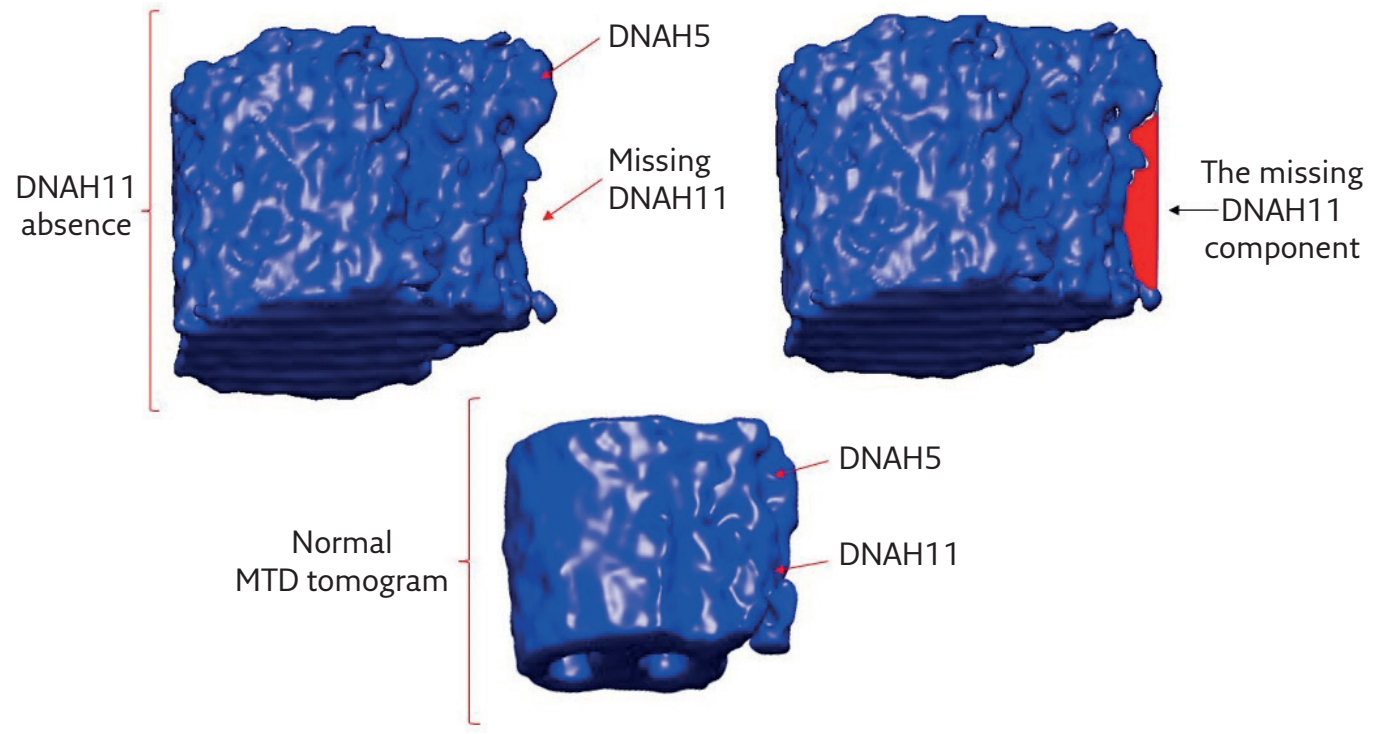

Figure 5 Tomogram from a patient with DNAH11 mutation showing loss of ODA protein proximally in first image, and where the missing protein would be located in second image in red. The third image shows normal outer dynein arm structure in a patient with microtubular disorganisation (MTD). 
Table 2 Known genes associated with defects in cilium structure seen on TEM or HSVM

\begin{tabular}{|c|c|}
\hline Defect in cilia & Associated genes \\
\hline ODA & $\begin{array}{l}\text { DNAH5, DNAH1 1, DNAI1, DNAI2, DNAL1, DNAH9, } \\
\text { NME8 }\end{array}$ \\
\hline IDA & DNAH1, TTC12, CFAP57 \\
\hline $\begin{array}{l}\text { Pre-assembly } \\
\text { factors }\end{array}$ & $\begin{array}{l}\text { DNAAF1, DNAAF2, DNAAF3, DNAAF4, DNAAF5, LRRC6, } \\
\text { ZYMND10, SPAG1, PIH1D3, C21ORF59, CFAP300 }\end{array}$ \\
\hline $\begin{array}{l}\text { MTD/central } \\
\text { complex }\end{array}$ & HYDIN, STK36 \\
\hline Radial spoke & RSPH1, RSPH4A, RSPH9, RSPH3, DNAJB13 \\
\hline $\begin{array}{l}\text { 96-nm axonemal } \\
\text { ruler/IDA }\end{array}$ & $C C D C 39, C C D C 40$ \\
\hline Nexin link/N-DRC & CCDC164, CCDC65, GAS8 \\
\hline RGMC & MCIDAS, CCNO, FOXJ1 \\
\hline Cytoplasmic & $R P G R$, OFD1, CFAP221 \\
\hline ODA docking & CCDC114, ARMC4, CCDC151, TTC25, MNS1, CCDC103 \\
\hline PCD spectrum & CFAP53 (CCDCD11), ENKUR, GAS2L2, LRRC56, NEK10 \\
\hline
\end{tabular}

ODA: outer dynein arm; IDA: inner dynein arm; MTD: microtubular disorganisation; $\mathrm{N}$-DRC: nexin-dynein regulatory complex; RGMC: reduced generation of multiple motile cilia. defect tend to be diagnosed earlier, despite having no association with situs abnormalities, because of a more severe respiratory phenotype and lower body mass index when compared with patients who have DNAH5 mutations [42].

Genotype-phenotype correlations will become increasingly useful when counselling and managing patients.

\section{Establishing a PCD diagnostic reference centre}

The growing complexity of securing a diagnosis across such a heterogeneous group of conditions has led to attempts to develop comprehensive guidelines. The two international consensus guidelines, despite their differences, have led to useful debate around how best to improve diagnostics for PCD across a diverse landscape where resources and expertise can be limiting factors. This is particularly true for emerging centres but also is relevant for existing diagnostic services. The pursuit of a diagnosis has moved on from a binary approach based on the presence or absence of a trademark TEM defect. Similarly, identifying biallelic mutations in PCD genes cannot currently confirm $>80 \%$ of cases and where negative, cannot exclude the diagnosis. However, despite the growing number of diagnostic tests and tools at our disposal, significant challenges remain in establishing a reference centre with the comprehensive array of clinical tests needed to optimise diagnostic accuracy (figure 1).

Where expertise and resources are limited, a pragmatic approach to developing a pathway towards a diagnosis is key. What is feasible will depend on human factors as much as specialised microscopes. Having the required electron, confocal and light microscopes is only useful if a ciliary biologist is available. The expertise to analyse and interpret ciliary defects requires high throughput of samples to acquire the skill sets to achieve accurate diagnosis across an array of subtle but consistent anomalies. Working in isolation is also difficult in a condition where cross-checking and quality control rely on a team approach. A network of experts who collaborate to provide shared expertise has arisen from successive PCD consortia where training schools, workshops and conferences have facilitated skill acquisition, collaboration and research (BEATPCD, BESTcilia and ERS task forces). As a result, partnerships have developed between reference centres and emerging services, resulting in improved access to diagnostics and driving research.

With all of this in mind, a diagnostic centre requires the ability to perform at least two tests to secure a firm or highly likely diagnosis. Ideally, this will include TEM and/or genetic testing. Since both tests leave around $15-30 \%$ of cases undiagnosed, additional tests are required where the phenotype is convincing. $\mathrm{nNO}$ and IF are ideal back-up investigations that can be established 
across a wider laboratory setting, in particular where a hub-and-spoke network model might be more practical. They are useful as screening tests in centres that can link into a reference centre for the required additional testing to confirm the diagnosis. HSVM remains the single most abnormal test in confirmed cases of PCD, making it potentially the most valuable tool in the diagnostic algorithm. However, it requires exposure to many cases and acquired expertise to develop the necessary skills in accurate interpretation. It should only be used in combination with TEM or genotyping where specific beat patterns should correlate with structural defects and specific mutations to underpin the accuracy of the diagnosis (table 2).

\section{Clinical management of respiratory ciliopathies}

Protocols for the management of PCD have largely been extrapolated from treatment programmes for $\mathrm{CF}$ with a respiratory centric focus. Both conditions have ciliary dysfunction-related retention of secretions, primary in PCD and acquired in CF, requiring airway clearance and treatment of chronic and recurrent infection. However, it is here that the overlap in these multisystem disorders ends with each requiring its own specialist multisystem approach for monitoring and treatment. The management of all respiratory ciliopathies will follow these strategies for patients with PCD, RGMC or syndromic ciliopathies in terms of respiratory, ear, nose and throat, and fertility medicine. However, some patients with ciliopathy syndromes will need additional comorbidity care focused on their specific needs.

\section{PCD management approach}

It should be noted that evidence-based medicine is in short supply for this condition, with clinical trials addressing treatments in non-CF bronchiectasis [52] or looking at small numbers of patients with PCD. The first randomised clinical trial studied the long-term use of azithromycin in patients with PCD and showed a significant reduction in infectious exacerbations [53]. There was no change in the other outcome measures (forced expiratory volume in $1 \mathrm{~s}$ and quality of life scores). The commonest pathogen found in sputum of patients with PCD is Haemophilus influenzae $[52,54]$. Others include Streptococcus pneumoniae, Staphylococcus aureus, Moraxella catarrhalis and Pseudomonas aeruginosa [55]. Prompt and more prolonged treatment of respiratory tract infections guided by microbiological findings is one of the pillars of managing PCD lung disease [52].

Mucolytic therapies are the second pillar and the first line of managing airway clearance. Here, practice between CF and PCD is beginning to diverge. In CF, sticky secretions are tackled using

\section{Self-assessment questions}

1 Nodal cilia are single motile cilia that are on cells within the embryonic node.
a. True
b. False

2 Which of the following statements are true?

a. There have been 50 genes identified as causing PCD.

b. There are multiple randomised clinical trials on treatment modalities in children with PCD.

c. Haemophilus is a common bacterium found in the sputum of patients with PCD.

d. All patients with suspected PCD require full panel of tests to confirm diagnosis.

3 Regarding respiratory ciliopathies, which of the following statements are true?

a. Patients with BBS have normal structural cilia.

b. HSVM in patients with sensory ciliopathies can show long, whip-like cilia with inclusion bodies at the tips, suggestive of an overlapping phenotype.

c. Patients with FOXJ1 mutation are unlikely to have respiratory involvement.

4 Which of the following tests are always positive in patients with PCD?
a. $\mathrm{nNO}$
b. TEM
c. Genetic variants

5 The European and American PCD diagnostic pathways are the same.
a. True
b. False

DNase, hypertonic saline or mannitol alone or in combination based on expert physiotherapist assessment. In PCD, experience has shown that hypertonic saline is most effective, loosening secretions and inducing cough to help clear secretions. Studies of the use of mucolytics are scarce and often include PCD in cohorts of patients with non-CF bronchiectasis [56, 57]. Studies such as that by PAFF et al. [57] are amongst the first to include only patients with PCD.

It is clear that despite the lack of clinical evidence, respiratory ciliopathies require specialised and directed comorbidity care. Common sense and sharing of expertise have led to a pragmatic approach but more randomised clinical trials are needed to establish truly effective therapies for this group of patients.

\section{Factors to consider for patients with more complex and syndromic ciliopathies}

For patients with syndromic ciliopathies, comorbidity care should be designed around their multiple needs. In the UK, specialist services have been designed around specific conditions, PCD, BBS, Alström 


\section{Suggested answers}

1. a.

2. a and c.

3. $a$ and $b$.

4. All are false.

5. b. syndrome and RP all being examples where a national approach to complex care needs has transformed clinical service provision and driven research programmes, leading to improved diagnosis and targeted therapeutic programmes. Patients with complex coronary heart disease with an underlying ciliopathy may be late to have their respiratory phenotype assessed as the severity of their other morbidities dominates the clinical picture. A wider group of syndromic ciliopathies with no dedicated care pathway may represent the most vulnerable group, where clinicians unfamiliar with the rarity of their condition and their complex needs may focus on dominating signs and symptoms, with the result that their progressive respiratory disease remains untreated, leading to relentless decline. As we continue to recognise the overlap in the motile and syndromic ciliopathies, greater collaborative working will be required to ensure that these patients are appropriately managed for their complex needs.

\section{Conclusion}

Respiratory ciliopathies are complex multifactorial pulmonary diseases demonstrating genetic heterogeneity with a common respiratory phenotype. PCD remains the best understood ciliopathy, with an established diagnostic pathway that has become well recognised. Over the last decade, new terminology is developing in response to a better understanding of the underlying cell biology and disease mechanisms. Some mutations affect assembly and transport of cilia proteins within the cell, resulting in effective aplasia rather than dyskinesia, and are now known as RGMC disorders within the wider respiratory ciliopathy class. So, it seems that PCD is just one of a wider group of respiratory ciliopathies where a better understanding of the heterogeneous nature of this group of conditions will lead to more accurate and descriptive terminology.

Affiliations

Reena Bhatt ${ }^{1}$, Claire Hogg 1,2

${ }^{1}$ Royal Brompton Hospital, London, UK. ${ }^{2}$ Paediatric Respiratory Medicine, Imperial College London, London, UK.

\section{Acknowledgements}

We would like to thank Amelia Shoemark (National PCD Diagnostic Service, Royal Brompton Hospital and Department of Molecular and Clinical Science, University of Dundee, Dundee, UK), Paul Griffen and Farheen Daudvohra (National PCD Diagnostic Service, Royal Brompton Hospital, London, UK) for their help in generating the diagnostic images in this article.

Conflict of interest

None declared.

References

1. Reiter JF, Leroux MR. Genes and molecular pathways underpinning ciliopathies. Nat Rev Mol Cell Biol 2017; 18: 533-547

2. Singla V, Reiter JF. The primary cilium as the cell's antenna: signalling at a sensory organelle. Science 2006; 313: 629-633.

3. Spassky N, Meunier A. The development and functions of multiciliated epithelia. Nat Rev Mol Cell Biol 2017; 18 423-436.

4. Hildebrandt F, Benzing T, Katsanis N. Ciliopathies. N Engl J Med 2011; 364: 1533-1543.

5. Goetz SC, Anderson KV. The primary cilium: a signalling centre during vertebrate development. Nat Rev Genet 2010 11: 331-344.

6. Mitchison HM, Valente EM. Motile and non-motile cilia in human pathology: from function to phenotypes. J Pathol 2017 . 241: 294-309.

7. Brooks ER, Wallingford JB. Multiciliated cells. Curr Bio/ 2014 24: R973-R982.

8. Pennekamp P, Menchen T, Dworniczak B, et al. Situs inversus and ciliary abnormalities: 20 years later, what is the connection? Cilia 2015; 4: 1

9. Leigh MW, Pittman JE, Carson JL, et al. Clinical and genetic aspects of primary ciliary dyskinesia/Kartagener syndrome. Genet Med 2009; 11: 473-487.
10. Mitchison HM, Shoemark A. Motile cilia defects in diseases other than primary ciliary dyskinesia: the contemporary diagnostic and research role for transmission electron microscopy. Ultrastruct Path 2017; 41: 415-427.

11. Lee JE, Gleeson JG. A systems-biology approach to understanding the ciliopathy disorders. Genome Med 2011; 3: 59

12. Milla CE. The evolving spectrum of ciliopathies and respiratory disease. Curr Opin Pediatr 2016; 28: 339-347.

13. Shah AS, Ben-Shahar Y, Moninger TO, et al. Motile cilia of human airway epithelia are chemosensory. Science 2009; 325 1131-1134.

14. Bloodgood RA. Sensory reception is an attribute of both primary cilia and motile cilia. J Cell Sci 2010; 123: 505-509.

15. Shoemark A, Dixon M, Beales PL, et al. Bardet Biedl Syndrome: Motile ciliary phenotype. CHEST 2015; 147: 764-770.

16. Bukowy-Bieryllo Z, Zietkiewicz E, Loges NT, et al. RPGR mutations might cause reduced orientation of respiratory cilia. Pediat Pulmonol 2013; 48: 352-363.

17. Moua T, Zand L, Hartman RP, et al. Radiologic and clinical bronchiectasis associated with autosomal dominant polycystic kidney disease. PLoS One 2014; 18: e93674.

18. Driscoll JA, Bhalla S, Liapis $\mathrm{H}$, et al. Autosomal dominan polycystic kidney disease is associated with an increased 
prevalence of radiographic bronchiectasis. CHEST 2008; 133 : 1181-1188.

19. Wallmeier J, Frank D, Shoemark A, et al. De novo mutations in FOXJ1 result in motile ciliopathy with hydrocephalus and randomisation of left/right body asymmetry. Am J Hum Genet 2019; 105: 1030-1039.

20. Kennedy M, Plant B. Primary ciliary dyskinesia and the heart: cilia breaking symmetry. CHEST 2014; 146: 1136-1138.

21. Best S, ShoemarkA, Rubbo B, et al. Risk factors for situs defects and congenital heart disease in primary ciliary dyskinesia. Thorax 2019; 74: 203-205.

22. Shoemark A, Frost E, Dixon M, et al. Accuracy of Immunofluorescence in the Diagnosis of Primary Ciliary Dyskinesia. Am J Respir Crit Care Med 2017; 196: 94-101.

23. Shoemark A, Hogg C. Electron Tomography of respiratory cilia. Thorax 2013; 68: 190-191.

24. Thomas L, Bouhouche K, Whitfield M, et al. TTC12 loss-offunction mutations cause primary ciliary dyskinesia and unveil distinct dynein assembly mechanisms in motile cilia versus flagella. Am J Hum Gene 2020; 106: 1-17.

25. Hirst RA, Jackson CL, Coles JL, et al. Culture of primary ciliary dyskinesia epithelial cells at air-liquid interface can alter ciliary phenotype but remains a robust and informative diagnostic aid. PLoS One 2014; 9: e89675.

26. Lucas JS, Barbato A, Collins SA, et al. European Respiratory Society guidelines for the diagnosis of primary ciliary dyskinesia. Eur Respir J 2017; 49: 1601090

27. Barbato A, Frischer T, Kuehni CE, et al. Primary ciliary dyskinesia: a consensus statement on diagnostic and treatment approaches in children. Eur Respir J 2009; 34: 1264-1276.

28. Shapiro AJ, Davis SD, Polineni D, et al. Diagnosis of primary ciliary dyskinesia. An official American Thoracic Society clinical practice guideline. Am J Respir Crit Care Med 2018; 197: e24-e39.

29. Shoemark A, Dell S, Shapiro A, et al. ERS and ATS diagnostic guidelines for primary ciliary dyskinesia: similarities and differences in approach to diagnosis. Eur Respir J 2019; 54: 1901066.

30. Behan L, Dimitrov BD, Kuehni CE, et al. PICADAR: a diagnostic predictive tool for primary ciliary dyskinesia. Eur RespirJ 2016; 47: 1103-1112

31. Shapiro AJ, Josephson M, Rosenfeld M, et al. Accuracy of nasal nitric oxide measurement as a diagnostic test for primary ciliary dyskinesia. A systematic review and meta-analysis. Ann Am Thorac Soc 2017; 14: 1184-1196.

32. Canciani M, Barlocco EG, Mastella G, et al. The saccharin method for testing mucociliary function in patients suspected of having primary ciliary dyskinesia. Pediatr Pulm 1988: 5: 210-214.

33. Michl R, Hentschel J, Fischer C, et al. Reduced nasal nitric oxide production in cystic fibrosis patients with elevated systemic inflammation markers. PLOS One 2013; 8: 1-8.

34. Shoemark A, Moya E, Hirst RA, et al. High prevalence of CCDC103 p.His154Pro mutation causing primary ciliary dyskinesia disrupts protein oligomerisation and is associated with normal diagnostic investigations. Thorax 2018; 73: 157-166

35. Harris A, Bhullar E, Gove K, et al. Validation of a portable nitric oxide analyzer for screening in primary ciliary dyskinesias. BMC Pulm Med 2014; 14: 18.

36. Rickard K, Leigh M, Davis S, et al. The NIOXVERO differentiates primary ciliary dyskinesia from healthy children using nasal nitric oxide measurements. CHEST 2017; 152: A594.

37. Rubbo B, Shoemark A, Jackson CL, et al. Accuracy of HighSpeed Video Analysis to Diagnose Primary Ciliary Dyskinesia. Chest 2019; 155: 1008-1017.

38. Jackson CL, Behan L, Collins SA, et al. Accuracy of diagnostic testing in primary ciliary dyskinesia. Eur Respir J 2016; 47: 837-848
39. Omran H, Loges NT. Immunofluorescence staining of ciliated respiratory epithelial cells. Methods Cell Biol 2009; 91: 123-133.

40. Olbrich $\mathrm{H}$, Schmidts $M$, Werner $\mathrm{C}$, et al. Recessive HYDIN mutations cause primary ciliary dyskinesia without randomization of left-right body asymmetry. Am J Hum Genet 2012; 91: 672-684

41. Shoemark A, Burgoyne T, Kwan R, et al. Primary ciliary dyskinesia with normal ultrastructure: three-dimensional tomography detects absence of DNAH11. Eur Respir J 2018; 51: 1701809

42. Lucas JS, Davis SD, Omran H, et al. Primary ciliary dyskinesia in the genomics age. Lancet Respir Med 2019; 8: 202-216.

43. Bustamante-Marin X, Shapiro A, Sears P, et al. Identification of genetic variants in CFAP221 as a cause of primary ciliary dyskinesia. J Hum Genet 2020; 65: 175-180.

44. Fassad MR, Shoemark A, le Borgne P, et al. C11 orf70 mutations disrupting the intraflagellar transport-dependent assembly of multiple axonemal dyneins cause primary ciliary dyskinesia. Am J Hum Genet 2018; 102: 956-972.

45. Vanaken GJ, Bassinet L, Boon M, et al. Infertility in an adult cohort with primary ciliary dyskinesia: phenotype-gene association. Eur Respir J 2017; 50: 1700314.

46. Boon $\mathrm{M}$, Wallmeier J, Ma L, et al. MCIDAS mutations result in a mucociliary clearance disorder with reduced generation of multiple motile cilia. Nat Commun 2014; 5: 4418.

47. Olbrich $\mathrm{H}$, Häffner $\mathrm{K}$, Kispert $\mathrm{A}$, et al. Mutations in DNAH5 cause primary ciliary dyskinesia and randomization of leftright asymmetry. Nat Genet 2002; 30: 143-144.

48. Loges NT, Antony D, Maver A, et al. Recessive DNAH9 lossof-function mutations cause laterality defects and subtle respiratory ciliary-beating defects. Am J Hum Genet 2018; 103: 995-1008.

49. Castleman VH, Romio L, Chodhari R, et al. Mutations in radial spoke head protein genes $R S P H 9$ and $R S P H 4 A$ cause primary ciliary dyskinesia with central-microtubular-pair abnormalities. Am J Hum Genet 2009; 84: 197-209.

50. Wirschell M, Olbrich $H$, Werner $C$, et al. The nexin-dynein regulatory complex subunit DRC1 is essential for motile cilia function in algae and humans. Nat Genet 2013; 45: 262-268

51. Sironen A, ShoemarkA, Patel M, et al. Sperm defects in primary ciliary dyskinesia and related causes of male infertility. Cell Mol Life Sci 2020; 77: 2029-2048.

52. Damseh N, Quercia N, Rumman N, et al. Primary ciliary dyskinesia: mechanisms and management. Appl Clin Genet 2017; 10: 67-74.

53. Kobbernagel HE, Buchvald FF, Haarman EG, et al. Efficacy and safety of azithromycin maintenance therapy in primary ciliary dyskinesia (BESTCILIA): a multicentre, double-blind, randomised, placebo-controlled phase 3 trial. Lancet Respir Med 2020; 8: 493-505.

54. Alanin MC, Nielsen KG, von Buchwald M, et al. A longitudinal study of lung bacterial pathogens in patients with primary ciliary dyskinesia. Clin Microbiol Infect 2015; 21: 1093.

55. Kobbernagel HE, Buchvald FF, Haarman EG, et al. Study protocol rationale and recruitment in a European multicentre randomized controlled trial to determine the efficacy and safety of azithromycin maintenance therapy for 6 months in primary ciliary dyskinesia. BMC Pulm Med 2016; 16: 104.

56. Kuehni CE, Goutaki M, Kobbernagel HE. Hypertonic saline in patients with primary ciliary dyskinesia: on the road to evidence-based treatment for a rare lung disease. Eur Respir J 2017; 49: 1602514.

57. Paff T, Daniels J, Weersink E, et al. A randomised controlled trial on the effect of inhaled hypertonic saline on quality of life in primary ciliary dyskinesia. Eur Respir J 2017; 49: 1601770. 